\title{
Efficient Feed Preheat Targeting for Distillation by Feed Splitting
}

\author{
Bharat F. Deshmukh ${ }^{a}$, Ranjan K. Malik ${ }^{b}$ and Santanu Bandyopadhyay ${ }^{a^{*}}$ \\ ${ }^{a}$ Energy Systems Engineering and Department of Mechanical Engineering \\ Indian Institute of Technology, Bombay, Powai, Mumbai 400 076, India \\ ${ }^{\mathrm{b}}$ Computer Aided Design Centre and Department of Chemical Engineering \\ Indian Institute of Technology, Bombay, Powai, Mumbai 400 076, India
}

\begin{abstract}
Thermal condition of the feed may be altered to reduce the reboiler duty. However, only a portion of the thermal energy given to the feed reduces the reboiler duty. By splitting the feed and altering the thermal condition of a part of the feed, it is possible to achieve reduction only in the reboiler duty $(100 \%$ preheat efficiency). Based on the thermodynamic analysis of a distillation column, a methodology is developed to target split fraction of the feed for preheating to obtain reduction only in the reboiler duty.
\end{abstract}

Keywords: energy conservation; feed preheating; distillation; pinch; IRS curves.

\section{Introduction}

Distillation is one of the common unit operations used in chemical industries. Though distillation is energy intensive, it is not energy efficient. Thermodynamic analysis of a distillation column is important for synthesizing and developing energy-efficient distillation processes. Bandyopadhyay et al. (1999) introduced a novel pair of temperature-enthalpy $(T-H)$ curves, known as the Invariant Rectifying-Stripping (IRS) curves for a distillation column. The IRS curves are invariant to the column configuration (i.e., feed location in the column and number of stages) and depend only on sharpness of separation as well as operating pressure of the column. They are useful for setting quantitative targets such as minimum energy requirement, appropriate feed location, proper feed preheating, scope for side-condensers/reboilers, as well as thermoeconomic optimisation of a distillation column.

Thermal condition of the feed is one of the important schemes for energy-efficient design of a distillation column. By exchanging heat with the bottom product or with any other available low-grade heat sources, thermal condition of the feed may be altered to reduce the reboiler duty. There exists an efficiency associated with feed preheating as a portion of the thermal energy given to the feed reduces the reboiler duty and the rest increases the condenser duty (Liebert, 1993). The IRS curves help in predicting simultaneously the decrease in reboiler duty, increase in condenser duty and the location of the feed for a given feed-preheat duty (Bandyopadhyay et al., 2003).

\footnotetext{
* Corresponding author: santanu@me.iitb.ac.in
} 
Splitting the feed into two streams and preheating only one fraction of the feed stream, it is possible to improve feed preheat efficiency up to 100\% (Wankat and Kessler, 1993; Fidkowski and Agrawal, 1995). Soave and Feliu (2002) had proposed the use of steadystate simulator to find iteratively the appropriate amount of split-fraction to achieve $100 \%$ preheat efficiency.

In this paper a methodology is developed, based on the IRS curves, to find the appropriate feed splitting to obtain $100 \%$ preheat efficiency. The proposed methodology is also applicable for non-ideal systems with tangent pinch. Based on the location of the pinch for non-ideal systems with tangent pinch, benefits of feed preheating are to be determined. It is observed that for certain systems, feed preheating cannot reduce the reboiler duty as it results in increasing condenser duty only ( $0 \%$ preheat efficiency).

\section{Invariant Rectifying-Stripping (IRS) Curves}

As a precursor to the basic theory, the generation procedure and physical significance of the IRS curves are briefly discussed below. Detailed derivations of the equations that are employed in this section are given in Bandyopadhyay et al. (2004).

\subsection{Generation of IRS curves}

A complex column with $n$ feeds may be decomposed into $n$ simple columns keeping the purity and enthalpy of the end products of the $i$-th column same as that of the original multiple-feed column. For the $i$-th decomposed column, the distillate flow-rate $\left(D_{i}\right)$, bottom flow-rate $\left(B_{i}\right)$, and enthalpy difference $\left(\Delta_{i}\right)$ may be determined through the overall mass, component, and energy balances $\left(\Delta_{\mathrm{i}} \equiv Q_{\mathrm{ri}}-Q_{\mathrm{ci}}=D_{\mathrm{i}} H_{\mathrm{D}}+B_{\mathrm{i}} H_{\mathrm{B}}-F_{i} H_{\mathrm{Fi}}\right)$. Let $H_{\mathrm{Ri}}$ be the minimum condensing load (enthalpy surplus) required to cause separation from $x$ to $x_{\mathrm{D}}$ in $i$-th column. Plot of enthalpy surplus as a function of the equilibrium temperature is termed the invariant rectifying (IR) curve. The overall mass, component, and energy balances for the rectifying section of the $i$-th column may be combined to obtain the following expression for $H_{\mathrm{Ri}}$.

$$
H_{\mathrm{Ri}}=D_{i}\left[H_{\mathrm{V}}\left(x_{\mathrm{D}}-x\right) /(y-x)-H_{\mathrm{L}}\left(x_{\mathrm{D}}-y\right) /(y-x)-H_{\mathrm{D}}\right]
$$

Similarly, consider the stripping section of a decomposed distillation column with $H_{\mathrm{Si}}$ denoting the minimum reboiling load required to cause separation from $x$ to $x_{\mathrm{B}}$. This enthalpy deficit is then plotted as a function of the equilibrium temperature to give the invariant stripping (IS) curve.

$$
H_{\mathrm{Si}}=B_{i}\left[H_{\mathrm{V}}\left(x-x_{\mathrm{B}}\right) /(y-x)-H_{\mathrm{L}}\left(y-x_{\mathrm{B}}\right) /(y-x)+H_{\mathrm{B}}\right]
$$

The invariance of the IRS curves for binary systems may be proven using Gibb's phase rule. The invariant property of the IRS curves does not hold rigorously for multicomponent systems. The pseudo-binary concept of light and heavy keys are employed to generate near-invariance IRS curves (Bandyopadhyay, 1999).

Dhole and Linnhoff (1993) described a procedure for generating a $T-H$ curve, called the column grand composite curve (CGCC), from a converged simulation of a distillation column. The anomaly in generation of CGCC was rectified through a feed stage correction procedure (Bandyopadhyay et al., 1998). CGCC is configuration dependent. It is possible to coalesce all the CGCCs into a pair of IRS curves (Bandyopadhyay et al., 1999). 


\subsection{Order and location of feeds}

The IR curve and the IS curve for any feed are not independent. Combining component, mass and energy balances around the feed stage, the relation between the IR curve and the IS curve at that feed stage can be obtained.

$H_{\mathrm{STi}}=H_{\mathrm{RTi}} \quad$ at every feed stage

where $H_{\mathrm{RTi}}\left(=H_{\mathrm{Ri}}+\Delta_{\mathrm{i}} / 2+\left|\Delta_{\mathrm{i}} / 2\right|\right)$ and $H_{\mathrm{STi}}\left(=H_{\mathrm{Si}}-\Delta_{\mathrm{i}} / 2+\left|\Delta_{\mathrm{i}} / 2\right|\right)$ are the enthalpy coordinates for the translated IR curve and translated IS curve, respectively.

Physically, equation (3) signifies the intersection of the $q$-line for a feed with the equilibrium curve on the $x-y$ diagram. The intersection of the $i$-th $q$-line with the equilibrium curve is independent of the intersections for the remaining feeds. The appropriate location for $i$-th feed may be determined in terms of temperature $\left(T_{\mathrm{Fi}}\right)$ by finding the intersection of the respective translated IRS curves. The appropriate order for the feeds may be determined by simply arranging the target temperatures (e.g., feed $F_{2}$ is at a higher temperature than feed $F_{1}$ in Figure 1a).

\subsection{Composite IRS curves and energy targets}

The portion of the translated IR curve below $T_{\mathrm{Fi}}$ and the portion of the translated IS curve above $T_{\mathrm{Fi}}$ may be defined as the active portions of the translated IRS curves for the $i$-th decomposed column and consequently the $i$-th feed. Composite IRS curves are generated for the complex column by simply adding the enthalpy coordinates of the active translated IRS curves corresponding to all the feeds. The composite IRS curves are shown in Fig. 1 for a typical two-feed column based on the translated IRS curves of the two decomposed columns. The composite IRS curves consist of $H_{\mathrm{RT} 1}+H_{\mathrm{RT} 2}, H_{\mathrm{ST} 1}+$ $H_{\mathrm{RT} 2}$, and $H_{\mathrm{ST} 1}+H_{\mathrm{ST} 2}$ (Figure 1a). In general, for a column with $n$ feeds, $(n+1)$ curves are to be drawn based on the active portions of the translated IRS curves of the $n$ decomposed columns. Thus, for section $i$ of an $n$-feed column,

$H_{\mathrm{A}}=\sum_{\mathrm{j}=1}^{n} H_{\mathrm{Aj}}=\sum_{\mathrm{j}=1}^{\mathrm{i}} H_{\mathrm{STj}}+\sum_{\mathrm{j}=\mathrm{i}+1}^{n} H_{\mathrm{RTj}} \quad i=0,1, \ldots, n$

The active portions of the composite IRS curves $\left(H_{\mathrm{A}}\right)$ may be circumscribed by a rightangled trapezium. The widths of the parallel sides of the trapezium at the top and bottom define the minimum energy targets for the reboiler and condenser, respectively (see Figure 1a). Figure 1a illustrates the case where one of the intersection points of the composite IRS curves determines the pinch. This is observed to be the case whenever the IRS curves of the individual decomposed columns are monotonic in nature. Note that the composite IRS curves for the complex column are, however, not monotonic.

\section{Feed Preheating Targets}

Let us consider the case of preheating the entire feed in a single-feed column. If $Q_{\mathrm{F}}$ is the amount of heat exchanged with the feed, then the overall energy balance may be combined to obtain (Bandyopadhyay et al., 2003)

$Q_{\mathrm{F}}=H_{\mathrm{RT}}\left\{T_{\mathrm{FP}}\right\}-H_{\mathrm{ST}}\left\{T_{\mathrm{FP}}\right\}$

where subscript $\mathrm{p}$ denotes quantities for the column with preheating. The important conclusion from the above equation is that the enthalpy difference between the 
translated IRS curves at a certain temperature $T_{\mathrm{FP}}$ specifies the amount of heat required to change the feed stage temperature from $T_{\mathrm{F}}$ to $T_{\mathrm{FP}}$. This is illustrated in Figure $1 \mathrm{~b}$.

For better geometric understanding, the invariant stripping curve translated horizontally by an additional amount $Q_{\mathrm{F}}$ as well as the trapezium for determining minimum reboiler and condenser loads after feed preconditioning are shown by dashed lines. It is observed on Figure $1 \mathrm{~b}$ that preheating has caused the minimum condenser duty to increase by $\delta Q_{\mathrm{c}}$ and the minimum reboiler duty to decrease by $-\delta Q_{\mathrm{r}}$. For systems with monotonic IRS curves, $\delta Q_{\mathrm{c}}=H_{\mathrm{RT}}\left\{T_{\mathrm{FP}}\right\}-H_{\mathrm{RT}}\left\{T_{\mathrm{F}}\right\}$ and $-\delta Q_{\mathrm{r}}=H_{\mathrm{ST}}\left\{T_{\mathrm{F}}\right\}-H_{\mathrm{ST}}\left\{T_{\mathrm{FP}}\right\}$. The efficiency associated with feed preheating may be defined as $\eta \equiv-\delta Q_{\mathrm{r}} / Q_{\mathrm{F}}$.

\subsection{Feed preheat targeting by feed splitting}

It is possible to split the feed into two parts and the thermal condition of one of the splitfractions may be altered. Let the split-fraction be $f$ that is preheated by $Q_{\mathrm{F}}$ and the other fraction $(1-f)$ be left unaltered. To generate the composite IRS curves and to address feed preheating effects, this two-feed column can now be decomposed into two single feed columns, as discussed earlier. Overall mass balance of the two decomposed columns reveals that the distillate and bottom flow-rates are related to the original column $\left[D_{1}=(1-f) D, D_{2}=f D, B_{1}=(1-f) B\right.$, and $\left.B_{2}=f B\right]$. Enthalpy difference of the decomposed columns reveals that $\Delta_{1}=(1-f) \Delta$ and $\Delta_{2 \mathrm{P}}=f \Delta-Q_{\mathrm{F}}$. Rectifying and stripping curves for the decomposed columns are calculated to be, $H_{\mathrm{RT} 1}=(1-f) H_{\mathrm{RT}}$, $H_{\mathrm{RT} 2 \mathrm{P}}=f H_{\mathrm{RT}}, H_{\mathrm{ST} 1}=(1-f) H_{\mathrm{ST}}$, and $H_{\mathrm{ST} 2 \mathrm{P}}=f H_{\mathrm{ST}}+Q_{\mathrm{F}}$.

Intersection of $H_{\mathrm{RT} 2 \mathrm{P}}$ and $H_{\mathrm{ST} 2 \mathrm{P}}$ indicates the location of the preheated split-fraction in the column (in temperature scale denoted as $T_{\mathrm{FP}}>T_{\mathrm{F}}$ ). Combining these equations, the composite IRS curves $H_{\mathrm{RT} 1}+H_{\mathrm{RT} 2 \mathrm{P}}\left(=H_{\mathrm{RT}}\right), H_{\mathrm{ST} 1}+H_{\mathrm{RT} 2 \mathrm{P}}\left(=(1-f) H_{\mathrm{ST}}+f H_{\mathrm{RT}}\right)$, and $H_{\mathrm{ST} 1}+H_{\mathrm{ST} 2 \mathrm{P}}\left(=H_{\mathrm{ST}}+Q_{\mathrm{F}}\right)$ for the complex column are obtained.

For $100 \%$ preheat efficiency, pinch should be controlled by the unaltered split-fraction. Maximum $Q_{\mathrm{F}}$ that can be utilized for $100 \%$ preheat efficiency should have two pinch points and both should lie on the same vertical line (points B and D as shown in Figure 2a). Point $D$ signifies the feed location point for the unaltered fraction of the feed. This controls the pinch point and hence the minimum energy required in the column. As a portion is preheated, a new feed location point is developed (point B). Whenever these two points have the same enthalpy value, minimum condenser duty (and hence the
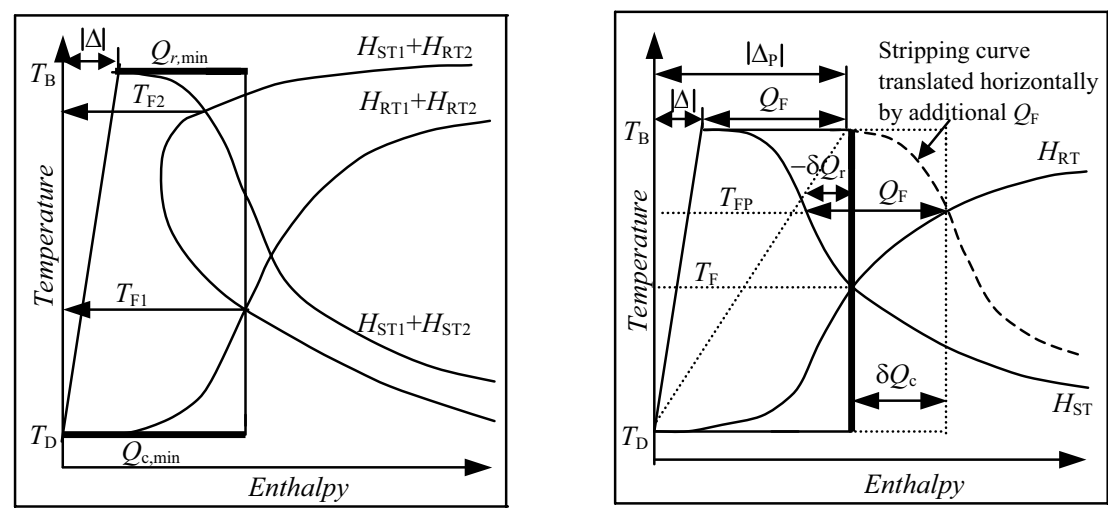

Figure 1. (a) Composite IRS curves for a two-feed column (b) Targeting feed preheat efficiency 

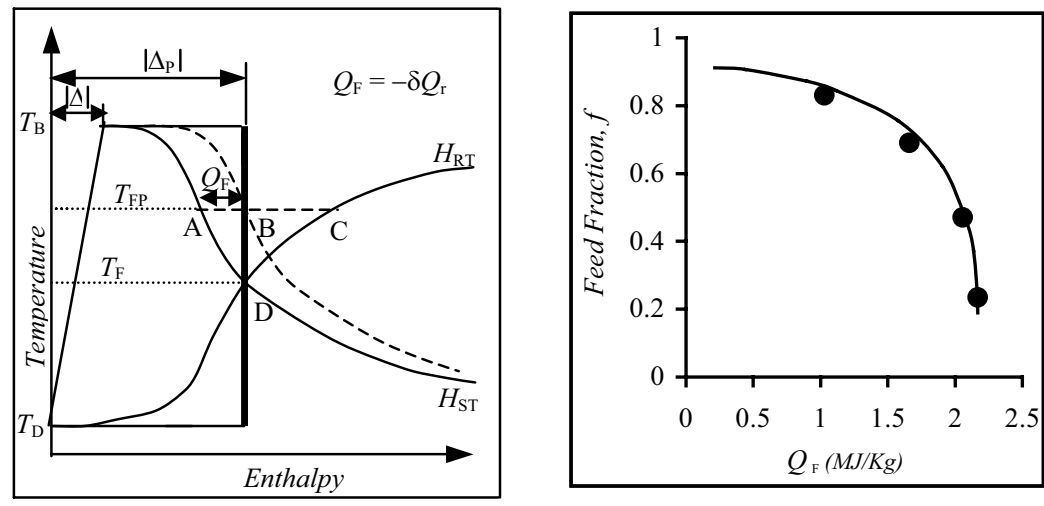

Figure 2. (a) Feed preheat targeting by feed splitting through composite IRS curves (b) Relation between feed split-fraction and preheat duty for the benzene-propane example

minimum reflux ratio) remains same as the original column. Constancy of the condenser load ensures $100 \%$ preheat efficiency. Criterion of two pinch points for $100 \%$ preheat efficiency translates into the following mathematical relations.

$H_{\mathrm{RT} 1}\left\{T_{\mathrm{F}}\right\}+H_{\mathrm{RT} 2 \mathrm{P}}\left\{T_{\mathrm{F}}\right\}=H_{\mathrm{ST} 1}\left\{T_{\mathrm{FP}}\right\}+H_{\mathrm{ST} 2 \mathrm{P}}\left\{T_{\mathrm{FP}}\right\}$

$H_{\mathrm{ST} 1}\left\{T_{\mathrm{FP}}\right\}+H_{\mathrm{RT} 2 \mathrm{P}}\left\{T_{\mathrm{FP}}\right\}=H_{\mathrm{ST} 1}\left\{T_{\mathrm{FP}}\right\}+H_{\mathrm{ST} 2 \mathrm{P}}\left\{T_{\mathrm{FP}}\right\}$

This may be simplified to

$Q_{\mathrm{F}}=H_{\mathrm{RT}}\left\{T_{\mathrm{F}}\right\}-H_{\mathrm{ST}}\left\{T_{\mathrm{FP}}\right\}=f\left[H_{\mathrm{RT}}\left\{T_{\mathrm{FP}}\right\}-H_{\mathrm{ST}}\left\{T_{\mathrm{FP}}\right\}\right]$

Rearranging this, the relation between the preheated split-fraction of the feed and the IRS curves can be expressed as

$f=\left[H_{\mathrm{RT}}\left\{T_{\mathrm{F}}\right\}-H_{\mathrm{ST}}\left\{T_{\mathrm{FP}}\right\}\right] /\left[H_{\mathrm{RT}}\left\{T_{\mathrm{FP}}\right\}-H_{\mathrm{ST}}\left\{T_{\mathrm{FP}}\right\}\right]$

Equations (8) and (9) can be interpreted through Figure 2a. If the feed location temperature of the preheated split fraction is raised to $T_{\mathrm{FP}}$, the amount of preheat required is shown by the line segment $\mathrm{AB}$ [equation (8)]. To achieve $100 \%$ preheat efficiency, the fraction of the split-feed can also be addressed form Figure 2a. This is given by the ratio of line segment $\mathrm{AB}$ to the line segment $\mathrm{AC}$ [equation (9)].

To design an energy-efficient distillation column with $100 \%$ preheat efficiency following methodology may be adopted: (a) From the base simulation of the column, IRS curves can be generated. (b) Location of the feed and the minimum energy required can now be calculated from the IRS curves. (c) For a given amount of feed preheat, the split-fraction and its location in the column can be targeted using equations (8) and (9). To demonstrate the potential of the described methodology, an example of benzenepropane has been considered. The column is operated at a constant pressure 15 bar with $99.9 \%$ purity on both products from the given feed $(100 \mathrm{~kg}-\mathrm{mol} / \mathrm{h}$ with $80 \%$ benzene at $20^{\circ} \mathrm{C}, 15$ bar). For this example, the simulations are performed using the DESIGN-II software. Variation of the feed preheat duty and the maximum split-fraction corresponding to $100 \%$ preheat efficiency, for this example is shown as firm line in Figure $2 \mathrm{~b}$. Detailed simulations, performed to verify these targets are also shown as dots in Figure 2b. Note that the simulation ranges form subcooled feed to superheated feed.

For non-ideal systems IRS curves are not monotonic. For systems with tangent pinch, 
IRS curves show a pinch point either in the rectifying or in the stripping section. In case the rectifying section holds the pinch, feed preheating will results in translating the IS curve to the right by $Q_{\mathrm{F}}$. Since the pinch point is still controlled by the rectifying curve, this implies that the feed preheat efficiency will be $100 \%$, even for the single feed. On the other hand, when the stripping section holds the pinch, feed preheating results in translating the IR curve to the left by $Q_{\mathrm{F}}$. Reboiler duty remains constant as the stripping section controls the pinch point. Preheating feed will results in increasing condenser duty only the corresponding feed preheat efficiency is $0 \%$, with or without feed splitting.

Composite IRS curves for a multi-feed column also exhibit nonmonotonicity. Depending on the location of the pinch controlling feed, other feeds will show either a $100 \%$ or $0 \%$ feed preheat efficiency.

\section{Conclusion}

Knowledge about the behaviour of a distillation column for different feed conditions is essential as it is important for integration of the column with the background process. It is also important for debottlenecking and utilization of the waste heat in the plant. Optimal thermal condition of the feed contributes significantly in increasing the thermodynamic efficiency of a cryogenic distillation column, since work (rather than heat) is being utilized in sub-ambient processes such as gas separation. In many industrial installations (e.g., naphtha stabilizer column) feed-bottom exchangers are used to reduce reboiler duty. Applications of the proposed methodology will decrease reboiler duty further without any capital investment.

In this paper a methodology is developed to find the appropriate feed splitting to obtain $100 \%$ preheat efficiency. The methodology helps in simultaneously targeting to find split fraction of feed for preheating and its location in the distillation column for a given amount of feed preheating. The proposed methodology may be applied to columns with side-products and for multicomponent systems (not discussed due to brevity). Based on these results a general principle can be devised for energy-efficient distillation process: feeds below the pinch should be cooled and feeds above it should be preheated.

\section{References}

Bandyopadhyay S., 1999, Energy targeting for optimisation of distillation processes, Ph.D. Thesis, Indian Institute of Technology, Bombay.

Bandyopadhyay S., R.K. Malik and U.V. Shenoy, 1998, Comp. Chem. Eng. 22, 1733.

Bandyopadhyay S., R.K. Malik and U.V. Shenoy, 1999, Comp. Chem. Eng. 23, 1109.

Bandyopadhyay S., R.K. Malik and U.V. Shenoy, 2003, Ind. Eng. Chem. Res. 42, 6851.

Bandyopadhyay S., M. Mishra and U.V. Shenoy, 2004, AIChE Journal, 50, 1837.

Dhole V.R. and B. Linnhoff, 1993, Comp. Chem. Eng, 17, 549.

Fidkowski Z.T. and R. Agrawal, 1995, Ind. Eng. Chem. Res. 34, 1287.

Liebert T., 1993, Hydrocarbon Process. 37.

Soave G. and J.A. Feliu, 2002, Appl. Thermal Eng. 22, 889.

Wankat P. and D.P. Kessler, 1993, Ind. Eng. Chem. Res. 32, 3061.

\section{Acknowledgements}

Last author is thankful to Industrial Research and Consultancy Centre, IIT Bombay, for providing partial financial support for this work. 